\title{
ON THE PROJECTIVE STRUCTURE OF
}

\section{A REAL HYPERSURFACE IN $\mathrm{C}_{n+1}$}

\author{
SHIING-SHEN CHERN
}

To Werner Fenchel on his 70th birthday.

Let $C_{n+1}$ be the complex number space of dimension $n+1$ with the coordinates $z^{1}, \ldots, z^{n+1}$. A real hypersurface $M$ is defined analytically by the equation

$$
r\left(z^{j}, \bar{z}^{j}\right)=0, \quad 1 \leqq j \leqq n+1,
$$

where $r$ is a real-valued function. We suppose $r$ to be smooth with $\operatorname{grad} r \neq 0$. For $n=1, \mathrm{~B}$. Segre observed that the corresponding equation

$$
r\left(z^{j}, a^{j}\right)=0,
$$

where $\bar{z}^{j}$ is replaced by arbitrary parameters $a^{j}$, defines a two-parameter family of curves in the complex plane whose invariants were first studied by $A$. Tresse in 1896 [5]. These invariants clearly provide local invariants of the hypersurface $M$ itself. But they do not give a complete system of invariants of $M$ under biholomorphic transformations in $\mathrm{C}_{n+1}$, as remarked by Elie Cartan [1]. The latter problem has been the object of a recent study by J. Moser and the author [3].

The purpose of this note is to carry out Segre's idea for general $n$ and relate it to the invariants given in [3]. Equation (2) defines an $(n+1)$ parameter family of hypersurfaces when $M$ is non-degenerate. Generalizing the work of Tresse, M. Hachtroudi showed that a projective connection can be defined intrinsically in the space of hyperplane elements of $C_{n+1}$ [4]. The definition is a generalization, by no means obvious, of the construction of classical projective geometry from the data of its hyperplanes; cf. also Chern [2], Yen [6] for a further generalization. We will show that the definition of Hachtroudi's connection is closely related to that of the connection in [3]. This study has the advantage that it works only with the variables $z^{j}$ and their holomorphic functions; the conjugate variables $\bar{z}^{j}$ are not involved. Could this fact be of significance for the results to play a rôle in abstract algebraic geometry?

* This work was partially supported by NSF grant GP-34785-X.

Received January 6, 1975. 


\section{The Equivalence Problem.}

We put $w=z^{n+1}$. The hypersurfaces (2) can be considered as the integral hypersurfaces of the completely integrable differential system

$$
d w-p_{\alpha} d z^{\alpha}=0, d p_{\alpha}-r_{\alpha \beta} d z^{\beta}=0,
$$

where $r_{\alpha \beta}=r_{\beta \alpha}$ are holomorphic functions of $z^{\alpha}, w, p_{\beta}$. (Throughout this paper small Greek indices will run from 1 to $n$ and the summation convention will be adopted.) The latter variables, i.e., $z^{\alpha}, w, p_{\beta}$, can be interpreted as the coordinates in the space of hyperplane elements in $\mathrm{C}_{n+1}$.

We allow biholomorphic changes of coordinates defined by

$$
\begin{aligned}
& z^{* \alpha}=z^{* \alpha}\left(z^{\beta}, w\right), \\
& w^{*}=w^{*}\left(z^{\beta}, w\right),
\end{aligned}
$$

and the transformation on $p_{\alpha}$ is given by expressing that $d w^{*}-p_{\alpha}{ }^{*} d z^{* \alpha}$ is a multiple of $d w-p_{\alpha} d z^{\alpha}$. It follows that the form $d w-p_{\alpha} d z^{\alpha}$ is defined up to a multiple and the sets of forms

$$
d w-p_{\alpha} d z^{\alpha}, d z^{\beta}
$$

and

$$
d w-p_{\alpha} d z^{\alpha}, d p_{\alpha}-r_{\alpha \beta} d z^{\beta}
$$

are each defined up to a linear transformation. Following the general procedure in studying equivalence problems, we set

where

$$
\begin{aligned}
& \omega=u\left(d w-p_{\alpha} d z^{\alpha}\right), \\
& \omega^{\alpha}=u_{\beta}^{\alpha} d z^{\beta}+u^{\alpha}\left(d w-p_{\beta} d z^{\beta}\right), \\
& \omega_{\alpha}=v_{\alpha}\left(d w-p_{\beta} d z^{\beta}\right)+v_{\alpha}^{\beta}\left(d p_{\beta}-r_{\beta \gamma} d z^{\nu}\right),
\end{aligned}
$$

$$
u, u_{\alpha}^{\beta}, v_{\alpha}^{\beta}, u^{\alpha}, v_{\alpha}
$$

are new variables satisfying

$$
u \neq 0, \operatorname{det}\left(u_{\alpha}^{\beta}\right) \neq 0, \operatorname{det}\left(v_{\alpha}^{\beta}\right) \neq 0 .
$$

Then the forms in (6) are invariant in the space of all the variables: the ones in (7), together with $z^{\alpha}, w, p_{\beta}$. Computing $\bmod \omega$, we find

The condition

$$
\begin{aligned}
d \omega & \equiv u d z^{\alpha} \wedge d p_{\alpha}, \\
i \omega^{\alpha} \wedge \omega_{\alpha} & \equiv i u_{\beta}^{\alpha} v_{\alpha}^{e} d z^{\beta} \wedge\left(d p_{\boldsymbol{e}}-r_{\boldsymbol{e \gamma}} d z^{\gamma}\right) .
\end{aligned}
$$

$$
d \omega \equiv i \omega^{\alpha} \wedge \omega_{\alpha}
$$


is therefore equivalent to

$$
u \delta_{\alpha}^{\beta}=i u_{\alpha}^{\gamma} v_{\gamma}^{\beta} .
$$

We suppose (8) fulfilled, and set

$$
d \omega=i \omega^{\alpha} \wedge \omega_{\alpha}+\omega \wedge \varphi,
$$

where $\varphi$ is defined up to the change

$$
\varphi \rightarrow \varphi+t \omega .
$$

We will take $t$ as another new variable. Our variables are now

$$
u(\neq 0), u_{\alpha}^{\beta}, u^{\alpha}, v_{\alpha}, t, z^{\alpha}, w, p_{\alpha},
$$

which are $(n+2)^{2}-1$ in number, the $v_{\alpha}^{\beta}$ being determined by (8), and we have the invariant forms $\omega, \omega^{\alpha}, \omega_{\alpha}, \varphi$. Our purpose is to show that it is possible to introduce

$$
(n+2)^{2}-1-(2 n+2)=n^{2}+2 n+1
$$

other invariant forms, characterized by intrinsic conditions, so that the totality forms an independent set.

Clearly we can write

$$
d \omega^{\alpha}=\omega^{\beta} \wedge \varphi_{\beta}^{\alpha}+\omega \wedge \varphi^{\alpha} .
$$

Lemma 1. The forms $\varphi_{a}^{\beta}$ in (12) can be so chosen that

$$
d \omega_{\alpha} \equiv \varphi_{\alpha}^{\beta} \wedge \omega_{\beta}+\omega_{\alpha} \wedge \varphi, \quad \bmod \omega .
$$

They are then determined up to additive terms in $\omega$.

In fact, exterior differentiation of (9) gives the equation

$$
i \omega^{\alpha} \wedge\left(-d \omega_{\alpha}+\varphi_{\alpha}^{\beta} \wedge \omega_{\beta}+\omega_{\alpha} \wedge \varphi\right)+\omega \wedge\left(-d \varphi+i \varphi^{\alpha} \wedge \omega_{\alpha}\right)=0 .
$$

It follows that

$$
-d \omega_{\alpha}+\varphi_{\alpha}^{\beta} \wedge \omega_{\beta}+\omega_{\alpha} \wedge \varphi \equiv 0, \bmod \omega, \omega^{\beta} .
$$

Since the system

$$
\omega=0, \omega_{\alpha}=0
$$

is completely integrable, the left-hand side of $(14 a)$ is also $\equiv 0, \bmod \omega, \omega_{\beta}$. Hence we have

$$
-d \omega_{\alpha}+\varphi_{\alpha}^{\beta} \wedge \omega_{\beta}+\omega_{\alpha} \wedge \varphi \equiv a_{\alpha \beta}{ }^{\gamma} \omega^{\beta} \wedge \omega_{\gamma}, \bmod \omega .
$$

Substituting into (14), we get

$$
a_{\alpha \beta}^{\gamma}=a_{\beta \alpha}{ }^{\gamma}
$$


Writing $\varphi_{\alpha}^{\beta}$ for $\varphi_{\alpha}^{\beta}-a_{\alpha \gamma}{ }^{\beta} \omega^{\gamma}$ fulfills the equation (13) and leaves (12) unchanged. The second statement in the lemma is immediate.

We shall therefore put

$$
d \omega_{\alpha}=\varphi_{\alpha}^{\beta} \wedge \omega_{\beta}+\omega_{\alpha} \wedge \varphi+\omega \wedge \varphi_{\alpha} .
$$

Using (14) we let

$$
d \varphi=i \omega^{\alpha} \wedge \varphi_{\alpha}+i \varphi^{\alpha} \wedge \omega_{\alpha}+\omega \wedge \psi,
$$

where $\psi$ is a new one-form. The forms $\varphi_{\alpha}{ }^{\beta}, \varphi^{\alpha}, \varphi_{\alpha}, \psi$ are determined up to the transformation

$$
\begin{aligned}
\varphi_{\alpha}^{\beta} & =\varphi_{\alpha}{ }^{* \beta}+b_{\alpha}{ }^{\beta} \omega, \\
\varphi^{\alpha} & =\varphi^{* \alpha}+b_{\beta}{ }^{\alpha} \omega^{\beta}+c^{\alpha} \omega, \\
\varphi_{\alpha} & =\varphi_{\alpha}{ }^{*}-b_{\alpha}{ }^{\beta} \omega_{\beta}+d_{\alpha} \omega, \\
\psi & =\psi^{*}+i\left(d_{\alpha} \omega^{\alpha}-c^{\alpha} \omega_{\alpha}\right)+e \omega .
\end{aligned}
$$

We shall determine the coefficients $b_{\alpha}^{\beta}, c^{\alpha}, d_{\alpha}, e$ by intrinsic conditions imposed on the exterior derivatives of the forms.

For this purpose we take the exterior derivatives of the equations (12) and (15). The resulting equations can be written

where we set

$$
\begin{gathered}
\omega^{\beta} \wedge \Phi_{\beta}^{\alpha}+\omega \wedge \Phi^{\alpha}=0, \\
\Phi_{\alpha}^{\beta} \wedge \omega_{\beta}-\omega \wedge \Phi_{\alpha}=0,
\end{gathered}
$$

$$
\begin{aligned}
\Phi_{\alpha}{ }^{\beta} & =d \varphi_{\alpha}{ }^{\beta}-\varphi_{\alpha}{ }^{\gamma} \wedge \varphi_{\gamma}{ }^{\beta}-i \omega_{\alpha} \wedge \varphi^{\beta}+i \varphi_{\alpha} \wedge \omega^{\beta}+i \delta_{\alpha}{ }^{\beta}\left(\varphi_{\sigma} \wedge \omega^{\sigma}\right)+\frac{1}{2} \delta_{\alpha}{ }^{\beta} \psi \wedge \omega, \\
\Phi^{\alpha} & =d \varphi^{\alpha}-\varphi \wedge \varphi^{\alpha}-\varphi^{\beta} \wedge \varphi_{\beta}{ }^{\alpha}+\frac{1}{2} \psi \wedge \omega^{\alpha}, \\
\Phi_{\alpha} & =d \varphi_{\alpha}-\varphi_{\alpha}{ }^{\beta} \wedge \varphi_{\beta}+\frac{1}{2} \psi \wedge \omega_{\alpha} .
\end{aligned}
$$

From (18) it follows that

where

$$
\Phi_{\alpha}^{\beta}=S_{\alpha \varrho}^{\beta \sigma} \omega^{\varrho} \wedge \omega_{\sigma}+\omega \wedge \psi_{\alpha}^{\beta},
$$

$$
S_{\alpha \varrho}^{\beta \sigma}=S_{\varrho \alpha}^{\beta \sigma}=S_{\alpha \varrho}^{\sigma \beta}
$$

and $\psi_{\alpha}^{\beta}$ is a one-form. Equation (18) then gives

$$
\begin{aligned}
& \Phi^{\alpha}=\omega^{\beta} \wedge{\psi_{\beta}}^{\alpha}+\omega \wedge \lambda^{\alpha}, \\
& \Phi_{\alpha}=\psi_{\alpha}{ }^{\beta} \wedge \omega_{\beta}+\omega \wedge \mu_{\alpha},
\end{aligned}
$$

where $\lambda^{\alpha}, \mu_{\alpha}$ are one-forms.

Applying the transformation (17) and denoting the new coefficients by asterisks, we get

$$
S_{\alpha \varrho}^{* \beta \sigma}=S_{\alpha \varrho}^{\beta \sigma}-i\left(\delta_{\varrho}{ }^{\sigma} b_{\alpha}^{\beta}+\delta_{\alpha}{ }^{\sigma} b_{\varrho}^{\beta}+\delta_{\varrho}^{\beta} b_{\alpha}{ }^{\sigma}+\delta_{\alpha}^{\beta} b_{\varrho}{ }^{\sigma}\right) .
$$


Putting

$$
S_{\alpha}^{\beta}=S_{\alpha \varrho}^{\beta Q}, S_{\alpha}^{* \beta}=S_{\alpha \varrho}^{* \beta Q},
$$

the contraction of $(23)$ gives

$$
S_{\alpha}^{* \beta}=S_{\alpha}^{\beta}-i(n+2) b_{\alpha}^{\beta}-i \delta_{\alpha}^{\beta} b_{e}^{e} .
$$

Lemma 2. The forms $\varphi_{\alpha}{ }^{\beta}$ are determined uniquely by the condition

$$
S_{\alpha}^{\beta}=0 \text {. }
$$

In fact, setting $S_{\alpha}^{* \beta}=0$ in (25), we find

$$
i(n+2) b_{\alpha}^{\beta}=S_{\alpha}^{\beta}-\frac{1}{2}(n+1)^{-1} \delta_{\alpha}^{\beta} S_{e}^{e} .
$$

From now on we suppose (26) to be fulfilled.

Exterior differentiation of (16) gives

$$
-i \omega^{\alpha} \wedge \Phi_{\alpha}+i \Phi^{\alpha} \wedge \omega_{\alpha}-\omega \wedge \Psi=0,
$$

where we set

$$
\Psi=d \psi-\varphi \wedge \psi-2 i \varphi^{\alpha} \wedge \varphi_{\alpha} .
$$

Exterior differentiation of the first equation of (19) gives

Contracting, we get

$$
\begin{aligned}
& d \Phi_{\alpha}{ }^{\beta}+\Phi_{\alpha}{ }^{\sigma} \wedge \varphi_{\sigma}{ }^{\beta}-\varphi_{\alpha}{ }^{\sigma} \wedge \Phi_{\sigma}{ }^{\beta}-i \omega_{\alpha} \wedge \Phi^{\beta}-i \Phi_{\alpha} \wedge \omega^{\beta} \\
&-i \delta_{\alpha}{ }^{\beta} \Phi_{\sigma} \wedge \omega^{\sigma}-\frac{1}{2} \delta_{\alpha}{ }^{\beta} \Psi \wedge \omega=0 .
\end{aligned}
$$

$$
d \Phi_{\alpha}{ }^{\alpha}-i(n+1) \Phi_{\sigma} \wedge \omega^{\sigma}-i \Phi^{\alpha} \wedge \omega_{\alpha}-\frac{1}{2} n \Psi \wedge \omega=0 .
$$

On the other hand, by (20) we have, as a result of (26),

$$
\Phi_{\alpha}^{\alpha}=\omega \wedge \psi_{\alpha}^{\alpha} \text {. }
$$

Substitution of this and (22) into (31) gives

whence

$$
(n+2) \psi_{\alpha}{ }^{\beta}+\delta_{\alpha}{ }^{\beta} \psi_{\ell}{ }^{\ell} \equiv 0, \quad \bmod \omega, \omega^{\ell}, \omega_{\sigma},
$$

$$
\psi_{\alpha}^{\beta} \equiv R_{\alpha \gamma}^{\beta} \omega^{\gamma}+T_{\alpha}^{\beta \gamma} \omega_{\gamma}, \quad \bmod \omega .
$$

We can therefore write $(20)$ as

$$
\Phi_{\alpha}^{\beta}=S_{\alpha \ell}^{\beta \sigma} \omega^{\ell} \wedge \omega_{\sigma}+R_{\alpha \gamma}^{\beta} \omega \wedge \omega^{\gamma}+T_{\alpha}^{\beta \gamma} \omega \wedge \omega_{\gamma} .
$$

Lemma 3. The forms $\varphi^{\alpha}$ and $\varphi_{\alpha}$ are determined uniquely by the conditions

$$
R_{\alpha \beta}^{\alpha \beta}=0, T_{\alpha \beta}^{\alpha \beta}=0 \text {. }
$$


ON THE PROJECTIVE STRUCTURE OF A REAL HYPERSURFACE IN $\mathrm{C}_{\boldsymbol{n}+1} \mathbf{7 9}$ When they are fulfilled, we have

and (22) can be written

$$
R_{\alpha \gamma}^{\beta}=R_{\gamma \alpha}^{\beta}, T_{\alpha}^{\beta \gamma}=T_{\alpha}^{\gamma \beta},
$$

$$
\begin{aligned}
& \Phi^{\alpha}=T_{\beta}^{\alpha \gamma} \omega^{\beta} \wedge \omega_{\gamma}+\omega \wedge \lambda^{\alpha}, \\
& \Phi_{\alpha}=R_{\alpha \gamma}^{\beta} \omega^{\gamma} \wedge \omega_{\beta}+\omega \wedge \mu_{\alpha} .
\end{aligned}
$$

In fact applying the transformation (17) to the first equation of (19), noticing that $b_{\alpha}^{\beta}=0$ and $\varphi_{\alpha}{ }^{\beta}$ are completely determined, we find

so that

$$
\begin{aligned}
& R_{\beta \gamma}^{*_{\alpha}}=R_{\beta \gamma}{ }^{\alpha}{ }-i \delta_{\gamma}{ }^{\alpha} d_{\beta}-\frac{1}{2} i \delta_{\beta}{ }^{\alpha} d_{\gamma}, \\
& T_{\beta}^{*_{\alpha \gamma}}=T_{\beta}{ }^{\alpha \gamma}-i \delta_{\beta}{ }^{\gamma} c^{\alpha}-\frac{1}{2} i \delta_{\beta}{ }^{\alpha} c^{\gamma},
\end{aligned}
$$

$$
\begin{aligned}
& R_{\alpha \gamma}^{*_{\alpha}}=R_{\alpha \gamma}^{\alpha}-i\left(\frac{1}{2} n+1\right) d_{\gamma}, \\
& T_{\alpha}^{*_{\alpha \gamma}}=T_{\alpha}^{\alpha \gamma}-i\left(\frac{1}{2} n+1\right) c^{\gamma} .
\end{aligned}
$$

Hence $c^{\gamma}$ and $d_{\gamma}$ can be determined to achieve (34).

With conditions (34) we have, from (33),

$$
\Phi_{\alpha}^{\alpha}=0 \text {. }
$$

Equations (28) and (31) then give

$$
\omega^{\alpha} \wedge \Phi_{\alpha}: \omega_{\alpha} \wedge \Phi^{\alpha}: \omega \wedge \Psi=1:-1:-2 i .
$$

This shows that $\Psi$ is of the form

$$
\Psi \equiv Q_{\alpha}{ }^{\beta} \omega^{\alpha} \wedge \omega_{\beta}, \quad \bmod \omega,
$$

and hence

$$
\begin{aligned}
& \omega^{\alpha} \wedge \Phi_{\alpha}=\frac{1}{2} i \omega \wedge Q_{\alpha}{ }^{\beta} \omega^{\alpha} \wedge \omega_{\beta}, \\
& \omega_{\alpha} \wedge \Phi^{\alpha}=-\frac{1}{2} i \omega \wedge Q_{\alpha}{ }^{\beta} \omega^{\alpha} \wedge \omega_{\beta} .
\end{aligned}
$$

From (22), (32), and (40) it follows that, $\bmod \omega$,

$$
0 \equiv \omega^{\alpha} \wedge \Phi_{\alpha} \equiv \omega^{\alpha} \wedge \psi_{\alpha}^{\beta} \wedge \omega_{\beta} \equiv \omega^{\alpha} \wedge\left(R_{\alpha \gamma}^{\beta} \omega^{\gamma}+T_{\alpha}^{\beta \gamma} \omega_{\gamma}\right) \wedge \omega_{\beta} .
$$

This relation implies (35), and hence also (36). This establishes Lemma 3.

From (36) and (40) we also get

$$
\begin{aligned}
& \omega \wedge \omega^{\alpha} \wedge\left(\mu_{\alpha}+\frac{1}{2} i Q_{\alpha}{ }^{\beta} \omega_{\beta}\right)=0, \\
& \omega \wedge \omega_{\beta} \wedge\left(\lambda^{\beta}+\frac{1}{2} i Q_{\alpha}{ }^{\beta} \omega^{\alpha}\right)=0 .
\end{aligned}
$$

We can therefore set

$$
\begin{aligned}
& \lambda^{\alpha}=-\frac{1}{2} i Q_{\beta}{ }^{\alpha} \omega^{\beta}+L^{\alpha \beta} \omega_{\beta}, \\
& \mu_{\alpha}=P_{\alpha \beta} \omega^{\beta}-\frac{1}{2} i Q_{\alpha}{ }^{\beta} \omega_{\beta},
\end{aligned}
$$


with

$$
L^{\alpha \beta}=L^{\beta \alpha}, P_{\alpha \beta}=P_{\beta \alpha} .
$$

Substituting into (36), we have the expressions

$$
\begin{aligned}
& \Phi^{\alpha}=T_{\beta}^{\alpha \gamma} \omega^{\beta} \wedge \omega_{\gamma}-\frac{1}{2} i Q_{\beta}{ }^{\alpha} \omega \wedge \omega^{\beta}+L^{\alpha \beta} \omega \wedge \omega_{\beta}, \\
& \Phi_{\alpha}=R_{\alpha \gamma}^{\beta} \omega^{\gamma} \wedge \omega_{\beta}+P_{\alpha \beta} \omega \wedge \omega^{\beta}-\frac{1}{2} i Q_{\alpha}^{\beta} \omega \wedge \omega_{\beta} .
\end{aligned}
$$

By using the second and third equations of (19) we immediately get the lemma:

Lemma 4. The form $\psi$ is completely determined by the condition

$$
Q_{\alpha}{ }^{\alpha}=0 \text {. }
$$

To find the expression for $\Psi$, we write down the equation obtained by exterior differentiation of (29), which is

$$
d \Psi-\varphi \wedge \Psi+2 i \Phi^{\alpha} \wedge \varphi_{\alpha}-2 i \varphi^{\alpha} \wedge \Phi_{\alpha}=0 .
$$

By (39) we set

$$
\Psi=Q_{\alpha}^{\beta} \omega^{\alpha} \wedge \omega_{\beta}+\omega \wedge \nu .
$$

Substituting this into (45) and making use of (43), we get

$$
\begin{aligned}
&\left\{d Q_{\alpha}{ }^{\beta}-Q_{Q}{ }^{\beta} \varphi_{\alpha}{ }^{Q}+Q_{\alpha}{ }^{\sigma} \varphi_{\sigma}{ }^{\beta}-2 Q_{\alpha}{ }^{\beta} \varphi+i \delta_{\alpha}{ }^{\beta} \nu+2 i T_{\alpha}{ }^{\alpha \beta} \varphi_{\sigma}-2 i R_{\sigma \alpha}{ }_{\alpha}{ }^{\sigma} \varphi^{\sigma}\right\} \omega^{\alpha} \wedge \omega_{\beta} \\
& \equiv 0, \bmod \omega .
\end{aligned}
$$

It follows that the expression between the braces is $\equiv 0 \bmod \omega, \omega^{\circ}, \omega_{\sigma}$. Contracting $\alpha, \beta$ and using (34), (35), (44), we conclude that $v$ is $\equiv 0$ $\bmod \omega, \omega^{\alpha}, \omega_{\beta}$. We can therefore put

$$
\Psi=Q_{\alpha}^{\beta} \omega^{\alpha} \wedge \omega_{\beta}+H_{\alpha} \omega \wedge \omega^{\alpha}+K^{\alpha} \omega \wedge \omega_{\alpha} .
$$

We summarize our results in the following theorem:

Theorem. Given in $\mathrm{C}_{n+1}$ an $(n+1)$-parameter family of hypersurfaces defined by the completely integrable differential system (3), there exist in the space of the variables (11), the same number of invariant differential forms

$$
\omega, \omega^{\alpha}, \omega_{\alpha}, \varphi, \varphi_{\alpha}^{\beta}, \varphi^{\alpha}, \varphi_{\alpha}, \psi,
$$

linearly independent, whose exterior derivatives are given by the "structure equations" (9), (12), (15), (16), (19), (29). The "curvature forms" $\Phi_{\alpha}{ }^{\beta}, \Phi^{\alpha}$, $\Phi_{\alpha}, \Psi$ have expressions given by (33), (43), (47), whose coefficients satisfy 
the symmetry relations (21), (34), (35), (42), (44). The forms (48) are completely determined by these conditions.

When $n=1, S_{\alpha \varrho}^{\beta \sigma}, R_{\alpha \gamma}^{\beta}, T_{\alpha}^{\beta \gamma}, Q_{\alpha}^{\beta}$ all vanish and the lowest-order invariants are $L^{11}, P_{11}$.

\section{Geometrical Construction.}

The tangent space $T_{z}$ at a point $z \in \mathrm{C}_{n+1}$ is a complex vector space of dimension $n+1$. We consider it as a part of a projective space $P T_{z}$ by adding to it a hyperplane at infinity. In turn $P T_{z}$ is considered as the quotient space of $V_{z}{ }^{*}=\left(V_{n+2}-\{0\}\right)_{z}$ by the action on $V_{n+2}-\{0\}$ by the multiplication of a non-zero complex number, where $V_{n+2}$ is the complex vector space of dimension $n+2$. To a point $\xi \in P T_{z}$ the components of the corresponding points of $V_{n+2}-\{0\}$, defined up to a non-zero factor, are called the homogeneous coordinates of $\xi$. In particular, we let a point $\left(y^{1}, \ldots, y^{n+1}\right) \in T_{z}$ to have the homogeneous coordinates $\left(y^{1}, \ldots, y^{n+1}, 1\right)$ and a vector $\left(v^{1}, \ldots, v^{n+1}\right)$, which can be considered as the difference of two points, to have the homogeneous coordinates $\left(v^{1}, \ldots, v^{n+1}, 0\right)$.

A projective frame in $P T_{z}$ consists of an ordered set $Z_{0}, Z_{1}, \ldots$, $Z_{n+1} \in V_{z}^{*}$, linearly independent and defined up to a common factor. On the other hand, a frame in $T_{z}$ consists of the origin $z$ and an ordered set of $n+1$ vectors. Using the above convention, to a frame in $T_{z}$ corresponds a uniquely determined projective frame in $P T_{z}$.

In the discussion of the last section the forms $\omega, \omega^{\alpha}$ constitute a coframe at $z \in C_{n+1}^{*}$. They determine uniquely a dual frame, and hence a projective frame $Z_{0}, \ldots Z_{n+1}$ in $P T_{z}$. As in [3], p. 260, we put

$$
\begin{gathered}
\pi_{0}{ }^{n+1}=2 \omega, \pi_{0}{ }^{\alpha}=\omega^{\alpha}, \pi_{n+1}{ }^{n+1}-\pi_{0}{ }^{0}=\varphi, \pi_{\alpha}{ }^{0}=-i \varphi_{\alpha}, \pi_{\alpha}{ }^{n+1}=2 i \omega_{\alpha}, \\
\pi_{n+1}{ }^{a}=\frac{1}{2} \varphi^{\alpha}, \pi_{\alpha}{ }^{\beta}-\delta_{\alpha}{ }^{\beta} \pi_{0}{ }^{0}=\varphi_{\alpha}{ }^{\beta}, \pi_{n+1}{ }^{0}=-\frac{1}{4} \psi
\end{gathered}
$$

Then it can be verified that the equations

$$
D Z_{A}=\pi_{A}^{B} Z_{B}, \quad 0 \leqq A, B \leqq n+1,
$$

define a projective connection. Except in notation this is essentially the one defined by M. Hachtroudi [4].

Finally we wish to make a remark on the relation of this connection with the one defined in [3]. We have chosen the notations so that the structure equations are identically the same. This implies that the projective connection underlies the connection in [3]. 


\section{BIBLIOGRAPHY}

1. E. Cartan, Sur la géométrie pseudo-conforme des hypersurfaces de deux variables complexes, I. Ann. Math. Pura Appl. (4) 11 (1932), 17-90, (or Oeuvres II, 2, 1231-1304);

II, Ann. Scuola Norm. Sup. Pisa (2) 1 (1932), 333-354 (or Oeuvres III, 2, 1217-1238).

2. S. S. Chern, A generalization of the projective geometry of linear spaces, Proc. Nat. Acad. Sci. USA, 29 (1943), 38-43.

3. S. S. Chern and J. K. Moser, Real hypersurfaces in complex manifolds, Acta Math. 133 (1974), 219-271.

4. M. Hachtroudi, Les espaces d'éléments à connexion projective normale, Hermann, Paris 1937.

5. B. Segre, I. Intorno al problema di Poincaré della rappresentazione pseudo-conforme, Rend. Acc. Lincei 13 (1931), 676-683; II. Questioni geometriche legate colla teoria delle funzioni di due variabili complesse, Rend. Semin. Mat. Roma 7 (1931).

6. Chih-ta Yen, Sur la connexion projective normale associée à un feuilletage du deuxième ordre, Ann. Math. Pura Appl. (4) 34 (1953). 55-94.

UNIVERSITY OF CALIFORNIA, BERKELEY, U.S.A. 